\title{
Power Assignment for $k$-Connectivity in Wireless Ad Hoc Networks
}

\author{
Xiaohua Jia* ${ }^{*}$ Dongsoo Kim ${ }^{\uparrow} \quad$ Sam Makki* Peng-Jun Wan ${ }^{\star} \quad$ Chih-Wei $\mathrm{Yi}^{\S}$
}

\begin{abstract}
The problem Min-Power $k$-Connectivity seeks a power assignment to the nodes in a given wireless ad hoc network such that the produced network topology is $k$-connected and the total power is the lowest. In this paper, we present several approximation algorithms for this problem. Specifically, we propose a $3 k$-approximation algorithm for any $k \geq 3$, a $(k+12 H(k))$-approximation algorithm for $k(2 k-1) \leq n$ where $n$ is the network size, a $(k+2[(k+1) / 2\rceil)$-approximation algorithm for $2 \leq k \leq 7$, a 6 -approximation algorithm for $k=3$, and a 9-approximation algorithm for $k=4$.
\end{abstract}

Index Terms-k-connectivity, power assignment, wireless ad hoc sensor networks

\section{INTRODUCTION}

One of the major concerns in ad hoc wireless networks is reducing node power consumption. In fact, nodes are usually powered by batteries of limited capacity. Once the nodes are deployed, it is very difficult or even impossible to recharge or replace their batteries in many application scenarios. Hence, reducing power consumption is often the only way to extend network lifetime. For the purpose of energy conservation, each node can (possibly dynamically) adjust its transmitting power between zero and its maximal transmission power. Throughout this paper, we use $V$ to denote the set of networking nodes and $\bar{p}(v)$ to denote the maximal transmission power of node $v$. In addition, we use $c(u v)$ to represent the power requirement for both node $u$ and

\footnotetext{
* School of Computing, Wuban University and Department of Computer Science, City University of Hong Kong. Email: jia@cs.cityu.edu.hk This work is supported in part by Hong Kong Research Grant Council under grant No. CityU 1149/04E.

${ }^{\dagger}$ Department of Electrical And Computer Engineering. Indiana Uiversity Purdue University, Indianapolis, IN 46202. Email: dskim@iupui.edu.

*Department of Electrical Engineering and Computer Science, University of Toledo. Email: kmakki@eng.utoledo.edu.

${ }^{\ddagger}$ Department of Computer Science, Illinois Institute of Technology, Chicago, Il 60616. Email: wan@cs.iit.edu. This work is partially supported by NSF CCR-0311174.

${ }^{8}$ Department of Computer Science, lllinois Institute of Technology Chicago, IL 60616. Email: yichihw@iit.edu.
}

node $v$ to establish a bidirectional link between $u$ and $v$. Then the wireless ad hoc network can be represented by a weighted graph $G=(V, E, c)$ with edges $u v$ whenever $c(u v) \leq \min (\bar{p}(u), \bar{p}(v))$. A power assignment to $V$ is a function $p: V \rightarrow R$ such that $0 \leq p(v) \leq \bar{p}(v)$ for each $v \in V$. The network topology produced by a power assignment is a spanning subgraph of $G$ with edges $u v$ whenever $c(u v) \leq \min (p(u), p(v))$.

Two or more paths in a (di)graph are independent if none of them contains an inner vertex of another. A (di)graph is $k$-connected if it contains at least $k$ independent paths between any pair of distinct vertices. By the well-known Menger's theorem, a (di)graph $k$ connected if and only if the deletion of any set of less than $k$ nodes leaves a connected (di)graph. The connectivity of (di)graph is defined to be the maximum $k$ for which it is $k$-connected. In this paper, we study the following Min-Power $k$-Connectivity problem: Find a power assignment with minimal total power to a given set of network nodes which produces a $k$-connected topology. An alternative description of this problem is as follows: For any subgraph $H$ of $G$, define $p_{H}(v)=$ $\max _{u v \in H} c(u v)$ for each $v \in V(H)$ and $p(H)=$ $\sum_{v \in V(H)} p_{H}(v)$; we call $p(H)$ the power (or power cost) of $H$. Since assigning $p(v) \geq p_{H}(v)$ is necessary to produce the subgraph $H$ and $p(v)>p_{H}(v)$ is just wasting power, the Min-Power $k$-Connectivity problem is equivalent to finding a $k$-connected spanning subgraph $H$ of $G$ with minimum $p(H)$.

For $k=1$ or 2 , the Min-Power $k$-Connectivity problem is NP-hard even with nodes located in a twodimensional Euclidean plane (see [2] for $k=1$ and [4] $k=2$ ). Thus, only polynomial-time approximation algorithms would be expected. It is strongly believed that for any positive integer $k$, the problem min-power for $k$-connectivity is NP-hard as well. Various approximation algorithms have been proposed for Min-Power $k$ Connectivity with different values of $k$. Table I summarizes the best-known approximation ratios published in the literature and the new approximation ratios obtained in this paper. More related works can be found from the 
cited works listed in Table I.

\begin{tabular}{|c||c|c|}
\hline$k$ & prior att & this paper \\
\hline \hline 1 & $5 / 3[3]$ & \\
\hline 2 & $4[4]$ & \\
\hline$k \geq 3$ & $8(k-1)[10]$ & $3 k$ \\
\hline$k(2 k-1) \leq n$ & & $k+12 H(k)$ \\
\hline $2 \leq k \leq 7$ & & $k+2\lceil(k+1) / 2\rceil$ \\
\hline 3 & - & 6 \\
\hline 4 & & 9 \\
\hline
\end{tabular}

TABLE I

BEST-KNOWN APPROXIMATION RATIOS AND THE APPROXIMATION RATIOS OBTAINED IN THIS PAPER. $H(k)$ IS THE $k$-TH HARMONIC NUMBER.

The remaining of this paper is organized as folFows. Section II defines relevant terms and notations. Section III presents several properties of the power cost. Section 4 gives a generic approach to approximation algorithms for Min-Power $k$-Connectivity, which leads to a $3 k$-approximation algorithm for any $k \geq 3$, a $(k+12 H(k))$-approximation algorithm for $k(2 k-1) \leq n$, and a $(k+2\lceil(k+1) / 2\rceil)-$ approximation algorithm for $2 \leq k \leq 7$. Section 7 presents a 6-approximation algorithm for Min-Power 3-Connectivity. Section 8 presents a 9-approximation algorithm for Min-Power 4-Connectivity.

\section{Definitions AND Notations}

Throughout this paper, we use $G=(V, E, c)$ to represent the wireless ad hoc network and assume that $G$ is $k$-connected. We denote by $O P T$ a min-power $k$-connected spanning subgraph of $G$, and by opt the power cost of $O P T$. For any $H \subseteq G$, we define $c(H)=\sum_{e \in E(H)} c(e)$; we call $c(H)$ the weight of $H$. We use $\vec{H}$ to represent the weighted graph obtained from $H$ be replacing every edge $u v$ of $G$ with two oppositely oriented arcs $u v$ and $v u$ with the same weight as the edge $u v$ in $H$. We also use $c(H)$ to denote the weight of $H$.

For any $D \subseteq \vec{G}$, we define $p_{D}(u)=\max _{u v \in D} c(u v)$ for each $u \in V(D)$ and $p(D)=\sum_{u \in V(D)} p_{D^{\prime}}(v)$; we call $p(D)$ the power of $D$. We also define $c(D)=$ $\sum_{e \in E(D)} c(e)$; we call $c(D)$ the weight of $D$. The out-degree of a node $v$ in $D$ is denoted by $\operatorname{deg}_{\bar{D}}(v)$. The maximum out-degree of $D$, i.e. $\max _{v \in V} \operatorname{deg}_{D}^{-}(v)$, is denoted by $\Delta^{-}(D) . D$ is called an in-branch if
$\Delta^{*}(D)=1$. An in-branch is called an in-arborescence if exactly one node, referred to as root, has zero outdegree. We use $\bar{D}$ to represent the undirected graph obtained from $D$ by ignoring the orientations of the links and then removing multiple edges between any pair of nodes.

For any $1 \leq i \leq k$, let be the $i$-nearest-neighbor subgraph of $G$ is a spanning subgraph of $G$, denoted by $G_{i}$, in which there is an edge between two nodes $u$ and $v$ if and only if either $u$ is one of the $i$ nearest neighbor of $v$ in $G$,or vice versa.

A (di)graph is said to be $k$-inconnected to a node $r$ if it contains $k$ independent paths to $r$ from any other vertex. Let $r$ be an arbitrary vertex of $D$. A min-weight spanning subdigraph of $D$ which is $k$-inconnected to $r$, if there is any, can be found in polynomial time by an algorithm of Frank and Tardos [8]. Gabow [9] has given an implementation of the Frank-Tardos algorithm that runs in time $O\left(k^{2} n^{2} m\right)$ where $n$ and $m$ are the number of vertices and number of arcs respectively of $D$. A minweight spanning subdigraph of $D$ which is $k$-inconnected to $s$ and in which the in-degree of $r$ is exactly $k$, if there is any, can also be found in polynomial time by applying the Frank-Tardos algorithm to an auxiliary graph [1].

The problem Min-Weight $k$-Connectivity problem takes as input a $k$-connected weighted graph $G$ and seek a $k$-connected spanning subgraph of $G$ with minimum weight. It is NP-hard for any fixed $k \geq 2$. A $k^{-}$ approximation algorithm was given in [11]. For $2 \leq$ $k \leq 7,\left\lceil\frac{k+1}{2}\right\rceil$-approximation algorithms were developed in [1] for $k=2,3$, in [7] for $k=4,5$, and in [11] for $k=6,7$. For the case $n \geq k(2 k-1)^{1}$, a $6 H(k)$-approximation algorithm was devised in [6], where $H(k)$ is the $k$-th harmonic number.

Let $H$ be a subgraph of a $k$-connected graph $G$. A subgraph $F$ of $G$ is called a $k$-connectivity augmentation to $H$ if $H \cup F$ is a $k$-connected spanning subgraph of $G$. The problem Min-Weight $k$-Connectivity Augmentation takes as input a $k$-connected weighted graph $G$ and a subgraph $H$ of $G$, and seeks a $k$-connectivity augmentation to $H$ in $G$ with minimum weight. We remark that any $\rho$-approximation algorithm $\mathcal{A}$ for Min-Weight $k$-Connectivity problem is also a $\rho$-approximation algorithm for Min-Weight $k$-Connectivity Augmentation problem. Indeed, for finding a $k$-connectivity augmentation to subgraph $H$ of $G=(V, E, c)$, we define a

\footnotetext{
${ }^{1}$ In the paper [6], $n$ is required to be at least $6 k^{2}$. The bound $n \geq k(2 k-1)$ is given by a recent work [14].
} 
new edge-weight function $c^{\prime}$ by $c^{\prime}(e)=0$ for any edge $e$ of $H$ and $c^{\prime}(e)=c(e)$ for any other edge $e$ of $G$. Then we apply $\mathcal{A}$ to the graph $\left(V, E, c^{\prime}\right)$ to obtain a $k$ connected spanning subgraph $H^{\prime}$. Then the graph $H^{\prime} \backslash H$ is a $k$-connectivity augmentation to $H$ and $c\left(H^{\prime} \backslash H\right)=$ $c^{\prime}\left(H^{\prime} \backslash H\right)$ is at most $\rho$ times of the minimum weight of a $k$-connectivity augmentation to $H$.

For any graph $H$ and any $S \subseteq V(H), S$ is said to be $k$-comnected in $H$ if $H$ contains $k$ independent paths between every pair of distinct vertices of $S$. Any graph with at least five vertices with minimal degree at least $k \geq 2$ contains a $k$-connected subset $S$ of four vertices [13]. In particular, $G_{4}$ contains a 4-connected subsets $S$ of four vertices.

\section{Basic Properties of Power Cos t}

In this section, we present some basic properties of power cost. The next lemma presents a relation between the power and the weight of a directed graph.

Lemma I: For any $D \subseteq \vec{G}, p(D) \leq c(D) \leq$ $\Delta^{-}(D) \cdot p(D)$.

Proof: Since

$$
\begin{aligned}
p(D) & =\sum_{u \in V(D)} p_{D}(u)=\sum_{u \in V(D)} \max _{u v \in E(D)} c(u v) \\
& \leq \sum_{u \in V(D)} \sum_{u v \in E(D)} c(u v)=\sum_{e \in E(D)} c(e)=c(D)
\end{aligned}
$$

the first inequality holds. Since

$$
\begin{aligned}
c(D) & =\sum_{e \in E(D)} c(e)=\sum_{u \in V(D)} \sum_{u v \in E(D)} c(u v) \\
& \leq \sum_{u \in V(D)} \operatorname{deg}_{D}^{-}(u) \max _{u v \in E(D)} c(u v) \\
& =\sum_{u \in V(D)} \operatorname{deg}_{\bar{D}}^{-}(u) p_{D}(u) \\
& \leq \Delta^{-}(D) \sum_{u \in V(D)} p_{D}(u)=\Delta^{-}(D) \cdot p(D),
\end{aligned}
$$

the second inequality holds.

The inequalities in Lemma 1 are tight. Indeed, if $D$ is an in-branch, then $p(D)=c(D)$; if $D$ is a uniformweighted out-star, then $c(D)=\Delta^{-}(D) \cdot p(D)$.

The next lemma presents a relation between the power and the weight of an undirected graph.
Lemma 2: For any $H \subseteq G, p(H) \leq 2 c(H)$. If $H$ is a forest, then $p(H)>c(H)$.

Proof: Since

$$
p(H)=p(\vec{H}) \leq c(\vec{H})=2 c(H),
$$

the first inequality holds.

For the second inequality, we first prove that for any tree $T, c(T)<p(T)$. Let $v$ be an arbitrary vertex of $T$. Orient $T$ to an in-arborescence $B$ rooted at $v$. Then

$$
c(T)=c(B)=p(B)<p(T) .
$$

Now let $T_{1}, T_{2}, \cdots, T_{l}$ be the tree components of $H$. Then

$$
c(H)=c\left(\bigcup_{i=1}^{l} T_{i}\right)=\sum_{i=1}^{l} c\left(T_{i}\right)<\sum_{i=1}^{l} p\left(T_{i}\right)=p(H) \text {. }
$$

Clearly, for any $H \subseteq G, p(\vec{H})=p(H)$ and $c(\vec{H})=2 c(H)$. The next lemma presents the relations between the weights (respectively, powers) of a directed graph $D$ and its undirected version $\bar{D}$.

Lemma 3: For any $D \subseteq \vec{G}$,

$$
\begin{aligned}
& c(D) \leq c(\bar{D}) \leq 2 c(D) . \\
& p(D) \leq p(\bar{D}) \leq\left(\Delta^{-}(D)+1\right) p(D) .
\end{aligned}
$$

Proof: Clearly, $c(D) \leq c(\bar{D}) \leq 2 c(D)$ and $p(D) \leq p(\bar{D})$. In the next, we prove that $p(\bar{D}) \leq$ $\left(\Delta^{-}(D)+1\right) p(D)$. For each node $v$, let $v^{\prime}$ be its farthest incoming neighbor. Then,

$$
\begin{aligned}
p_{\bar{D}}(v) & =\max \left\{p_{D}(v), c\left(v v^{\prime}\right)\right\} \\
& \leq \max \left\{p_{D}(v), p_{D}\left(v^{\prime}\right)\right\} \\
& \leq p_{D}(v)+p_{D}\left(v^{\prime}\right) .
\end{aligned}
$$

In order to bound $p(\bar{D})$, we introduce the following charging mechanism: each node $v$ imposes a charge of $p_{D}\left(v^{\prime}\right)$ on $v^{\prime}$. Then $p(\bar{D})$ is at most $p(D)$ plus the total charges imposed by all nodes. Since the out-degree of $D$ is $\Delta^{-}(D)$, each node receives charges from at most $\Delta^{-}(D)$ other nodes. Thus the total charge imposed on a node $u$ is at most $\Delta^{-}(D) p_{D}(u)$. So the total charges imposed by all nodes is at most $\Delta^{-}(D) p(D)$. This implies that $p(\bar{D}) \leq\left(\Delta^{-}(D)+1\right) p(D)$.

The inequalities in Lemma 1 are tight. Indeed, if $D$ is a uniform-weighted directed cycle, then $c(D)=c(\bar{D})$ and $p(D)=p(\bar{D})$; if $D$ is a uniform-weighted out-star, then $p(\bar{D})=\left(\Delta^{-}(D)+1\right) \cdot p(D)$; if for any pair of point $u$ and $v$, either both $u v$ and $v u$ are in $D$, or neither of them is in $D$, then $c(\bar{D})=2 c(D)$. 


\section{IV. $k$-CONNECTIVITY}

Let $\mathcal{A}$ be any approximation algorithm for Min-Weight $k$-Connectivity. We propose the following two-phased approximation algorithm for Min-Power $k$-Connectivity: Phase 1 constructs the $(k-1)$-nearest-neighbor graph $G_{k-1}$. Phase 2 applies $\mathcal{A}$ to find a $k$-connectivity augmentation $F$ to $G_{k-1}$ and outputs $G_{k-1} \cup F$. Its approximation ratio is given by the following theorem.

Theoren 4: Let $\mathcal{A}$ be any $\rho$-approximation algorithm for Min-Weight $k$-Connectivity . Let $F$ be the $k$ connectivity augmentation to $G_{k-1}$ produced by the algorithm $A$. Then $G_{k-1} \cup F$ is $k$-connected and $p\left(G_{k-1} \cup F\right) \leq(k+2 \rho)$ opt.

The proof of Theorem 4 shall be based on the two lemma below. The first lemma gives an upper bound on the power cost of the $i$-nearest-neighbor graph $G_{i}$.

Lemma 5: For any $1 \leq i \leq k, p\left(G_{i}\right) \leq(i+1)$ opt.

Proof: Let $D_{i}$ be the digraph in which there is an arc from $u$ to $v$ if and only if $v$ is one of the $i$ nearest neighbors of $u$. Then the maximum out-degree of $D$ is exactly $i$ and $G_{i}=\overline{D_{i}}$. Since the minimum degree of $O P T$ is at least $k$, for every node $v, p_{D_{i}}(v) \leq p_{O P T}(v)$ for every node $v$. Thus, $p\left(D_{i}\right) \leq p(O P T)=$ opt. By Lemma 3,

$$
p\left(G_{i}\right) \leq(i+1) p\left(D_{i}\right) \leq(i+1) \text { opt } .
$$

The second lemma gives an upper bound on the weight of a minimum-weighted $k$-connectivity augmentation to a graph with minimum degree at least $k-1$.

Lemma 6: Let $H$ be a spanning subgraph of $G$ with minimum degree at least $k-1$. Then the minimum weight of a $k$-connectivity augmentation to $H$ is less than opt.

Proof: Since $O P T$ is $k$-connected, it contains a $k$-connected augmentation to $H$. Let $F \subset O P T$ be a minimum-weighted $k$-connectivity augmentation to $H$. We first claim that $F$ is a forest. Assume that $F$ is not a forest. Then $F$ contains a cycle $C$. Since $F$ has minimum weight, every edge $e$ of $F$ is critical in $H \cup F$ with respect to $k$-connectivity, in other words, $(H \cup F) \backslash e$ is not $k$-connected. By Mader's theorem on "cycle of critical edges" [12], $C$ contains a node of degree $k$ in $H \cup F$. On the other hand, every node of $C$ is incident to two edges of $C$ and to at least $k-1$ edges of $H$. This means that the degree of every node of $C$ is at least $k+1$, which is a contradiction. Therefore, $F$ must be a forest. By Lemma 2,

$$
c(F)<p(F) \leq p(O P T)=o p t .
$$

So, the lemma follows.

Now we ready to prove Theorem 4 . The $k$-connectivity of $G_{k-1} \cup F$ is trivial since $F$ since $k$-connectivity augmentation to $G_{k-1}$. By Lemma $6, c(F)<\rho \cdot o p t$, which implies that $p(F)<2 \rho$ opt. By Lemma 5 , $p\left(G_{k-1}\right) \leq k \cdot$ opt. Therefore

$$
p\left(G_{k-1} \cup F\right) \leq p\left(G_{k-1}\right)+p(F)<(k+2 p) o p t .
$$

By choosing the algorithm by Kortsarz and Nutov [11] as $\mathcal{A}$, we obtain a $3 h$-approximation algorithm. For $n \geq$ $k(2 k-1)$, by choosing the approximation algorithm by Cheriyan et al. [6] as $\mathcal{A}$, we obtain a approximation ratio is $(k+12 \ln k)$-approximation algorithm. For $3 \leq k \leq$ 7 , by choosing the algorithms in [1], [7], [11] as $\mathcal{A}$, we get $\left(k+2\left\lceil\frac{k+1}{2}\right\rceil\right)$-approximation algorithms. However, for $k=3$ and 4 , even better approximation algorithms are possible, we are presented in the next two sections.

\section{3-CONNECTIVITY}

In this section, we present a 6 -approximation algorithm for Min-Power 3-Connectivity. It is motivated by the following theorem.

Theorem 7: Let $D$ be the min-weighted 3 inconnected spanning subdigraph of $\vec{G}$ in which the in-degree of the (unique) node without outgoing links is exactly 3 . Then, $\bar{D}$ is 3 -connected and $p(\bar{D})<6 \cdot$ opt.

Proof: The 3-connectivity of $\bar{D}$ follows Corollary 3.2 in [1]. Next, prove that $p(\bar{D})<6 \cdot$ opt. Two or more rooted spanning trees of a graph $G$ having the same root $r$ are said independent if for each vertex $v$ of $G$ the (unique) paths between $v$ and $r$ along these spanning trees are independent. It was proved independently by Cheriyan and Maheshwari [5] and Zehavi and Itai [15] that any 3-connected graph contains three independent spanning trees rooted at any common vertex. Let $T_{i}, 1 \leq$ $i \leq 3$, be three independent spanning trees of $O P T$ rooted at a common vertex $r$. For $1 \leq i \leq 3$, let $B_{i}$ be the in-branching obtained by orienting $T_{i}$ toward $r$. 
Then $\bigcup_{i=1}^{3} B_{i}$ is a 3 -inconnected digraph in which the in-degree of $r$ is exactly 3 . Therefore,

$c(D) \leq c\left(\bigcup_{i=1}^{3} B_{i}\right)=\sum_{i=1}^{3} c\left(B_{i}\right)=\sum_{i=1}^{3} p\left(B_{i}\right)<3 \cdot o p t$.

By Lemma 2 and Lemma 3,

$$
p(\bar{D}) \leq 2 c(\bar{D}) \leq 2 c(D)<6 \cdot o p t .
$$

Based on Theorem 7, we propose the following approximation algorithm for Min-Power 3-Connectivity: First apply the algorithm given in [1] to produce a minweighted 3 -inconnected spanning subdigraph $D$ of $\vec{G}$ in which the in-degree of the root is exactly 3 , and then output the graph $\bar{D}$. By Theorem 7, its approximation ratio is at most 6 .

\section{4-CONNECTIVITY}

In this section, we present a 9-approximation algorithm for Min-Power 4-Connectivity. The following auxiliary graph $G^{+}$will be used by the algorithm. Let $S$ be a set of four vertices in $V$ which is 4 -connected in $G_{4}$, whose existence is ensured by [13]. Then, $G^{+}$is the graph $\left(V^{+}, E^{+}, c^{+}\right)$in which $V^{+}=V \cup\{r\}$ for some vertex $r \notin V, E^{+}=E \cup\{r s: s \in S\}$, and

$$
c^{+}(e)= \begin{cases}0, & \text { if } e \in E\left(G_{4}\right) \cup\{r s: s \in S\} \\ c(e), & \text { otherwise. }\end{cases}
$$

A minimum-weighted 4-inconnected spanning subdigraph of $\overrightarrow{G^{+}}$rooted at $r$ has the following property.

Theorem 8: Let $D$ be a minimum-weighted 4inconnected spanning subdigraph of $\overrightarrow{G^{+}}$rooted at $r$. Then, $G_{4} \cup(\bar{D}-r)$ is 4-connected and $p\left(G_{4} \cup(\vec{D}-r)\right) \leq 9 \cdot$ opt.

Proof: We first prove the 4-connectivity of $G_{4} \cup$ $(\bar{D}-r)$. Let $C$ be any subset of $V$ with at most three vertices. Since $|S|=4, S \backslash C$ is nonempty, and since $S$ is 4 -connected in $G_{4}, S \backslash C$ is connected in $G_{4}-C$. Now let $v$ be any vertex in $V \backslash(C \cup S)$. Since $\bar{D}$ is 4 inconnected to $r$, there are fousr independent paths from $v$ to $r$ in $\bar{D}$, and all these four paths must path through a distinct vertex of $S$. Thus, there is still one at least one path in $(D-r)-C$ from $v$ to some node in $S \backslash C$. Hence, $\left(G_{4} \cup(\bar{D}-r)\right)-C$ remains connected. This implies that $G_{4} \cup(\bar{D}-r)$ is 4-connected.

Next we prove that $p\left(G_{4} \cup(\bar{D}-r)\right) \leq 9 \cdot$ opt. Let $F$ be a minimum-weighted 4-connectivity augmentation to $G_{4}$, and $H$ denote the graph $G_{4} \cup F \cup\{r s: s \in S\}$. Then by Lemma 6. $c(F)<$ opt. Since $G_{4} \cup F$ is 4 connected, $H$ is a 4-connected spanning subgraph of $G^{+}$, and consequently, $\vec{H}$ is a 4 -inconnected spanning subdigraph of $\overrightarrow{G^{+}}$rooted at $r$. Thus,

$$
c^{+}(D) \leq c^{+}(\vec{H})=2 c^{+}(H)=2 c(F)<2 o p t .
$$

Hence,

$$
c\left((\bar{D}-r) \backslash G_{4}\right)=c^{+}(\bar{D}) \leq c^{+}(D)<2 o p t .
$$

By Lemma 2, we have

$$
p\left((\bar{D}-r) \backslash G_{4}\right) \leq 2 c\left((\bar{D}-r) \backslash G_{4}\right)<4 \text { opt } .
$$

By Lemma 5, $p\left(G_{4}\right) \leq$ 5opt. So,

$p\left(G_{4} \cup(\bar{D}-r)\right) \leq p\left(G_{4}\right)+p\left((\bar{D}-r) \backslash G_{4}\right)<9 o p t$.

Based on Theorem 8, we propose the following approximation algorithm for Min-Power 4-Connectivity: First construct $G_{4}$ and find a 4-connected subset $S$ of four nodes by exhaustive search. Then construct the graph $G^{+}$and $\overrightarrow{G^{+}}$, and then apply Gabow's implementation [9] of Frank and Tardos algorithm [8] to produce a minimum-weighted 4-inconnected spanning subdigraph $D$ of $\overrightarrow{G^{+}}$rooted at $r$. Finally, output the graph $G_{4} \cup(\bar{D}-r)$. By Theorem 8, the approximation ratio of this approximation algorithm is at most 9 .

\section{CONCLUSION}

The problem Min-Power $k$-Connectivity seeks a power assignment to the nodes in a given wireless ad hoc network such that the produced network topology is $k$-connected and the total power is the lowest. In this paper, we present several approximation algorithms for this problem. Specifically, we give a $3 k$-approximation algorithm for any $k \geq 3$, a $(k+12 H(k))$-approximation algorithm for $k(2 k-1) \leq n$ where $n$ is the network size, a $(k+2\lceil(k+1) / 2\rceil)$-approximation algorithm for $2 \leq k \leq 7$, a 6-approximation algorithm for $k=3$, and a 9 -approximation algorithm for $k=4$. All these approxination results do not depend on the power attenuation characteristics affected by the deployment environtment. It would be interesting to know if the geometric nature of the power attenuation can be exploited to develop better and/or simpler approximation algorithms for Min-Power $k$-Connectivity. 


\section{REFERENCES}

[1] V. Auletta, Y. Dinitz, Z. Nutov, and D. Parente. A 2approximation algorithm for finding an optimum 3-vertexconnected spanning subgraph. J. Algorithms 31, pp. 21-30, 1999.

[2] D.M. Blough. M. Leoncini. G. Resta, and P. Santi, On the Symmetric Range Assignment Problem in Wireless Ad Hoc Networks, Proc. 2nd IFIP International Conference on Theoretical Computer Science. Montreal. August 2002.

[3] G. Calinescu, I. Mandoiu, and A. Zelikovsky. Symmetric Connectivity with Minimum Power Consumption in Radio Networks, submitted for journal publication, preliminary results appeared in Proc. 2nd IFIP International Conference on Theoretical Computer Science, 2002. pp. 119-130.

[4] G. Calinescu. P.-J. Wan. High Connectivity with Minimum Total Power in Wireless Ad Hoc Netowrks, Ad Hoc Now: 2003.

[5] J. Cheriyan and S.N. Maheshwari, Finding nonseparating induced cycles and independent spanning trees in connected graphs. J. Algorithms 9:507-537, 1988.

[6] J.Cheriyan, S.Vempala and A.Vetta, Approximation algorithms for minimum-cost $k$-vertex connected subgraphs, Proc. 34th Ann. ACM STOC. pp. 306-312. May 2002.

[7] Y. Dinitz, and Z. Nutov, A 3-Approximation Algorithm for Finding Optimum 4,5-Vertex-Connected Spanning Subgraphs, Joumal of Algorithms 32, 31-40, 1999.

[8] A. Frank and E. Tardos, An application of submodular flows, Linear Algebra and its Applications, vol. 114/115, pp. 329-348, 1989.

[9] H.N. Gabow, A representation for crossing set families with applications to submodular flow problems, in Proc. $4 \mathrm{th} \mathrm{ACM}$ SIAM Symposium on Discrete Algorithns, pp. 202-211, Austin, TX, 1993.

[10] M. T. Hajiaghayi, N. Immorlica, V. S. Mirrokni, Power optimization in fault-tolerant topology control algorithms for wireless multi-hop networks, Proceedings of the 9th annual international conference on Mobile computing and networking, Pages: 300 - 312, 2003.

[11] G.Kortsarz and Z. Nutov, Approximating node connectivity problems via set covers, Third International Workshop on Approximation Algorithms for Combinatorial Optimization, (APPROX 2000), Springer. LNCS 1913, 2000, pp. 194-205.

[12] W. Mader, Ecken vom Grad $n$ in minimalen $n$-fach zusammenhängenden Graphen, Arch. Math. 23 (1972). 219-224.

[13] W. Mader: Degree and local connectivity in finite graphs. Recent advances in graph theory (Proc. Second Czechoslovak Sympos.. Prague, 1974). Academia, Prague (1975), 341-344.

[14] W. Mader, On $k$-con-Critically $n$-Connected Graphs, Journal of Combinatorial Theory, Series B 86, 296-314 (2002).

[15] A. Zehavi and A. Itai, Three Tree-Paths. J. of Graph Theory, vol. 13(2). 177-188, (1989). 